\title{
Analysis of a HBV Model with Diffusion and Time Delay
}

\author{
Noé Chan Chí, Eric ÁvilaVales, and Gerardo García Almeida
}

Facultad de Matemáticas, Universidad Autónoma de Yucatán, Anillo Periférico Norte, Tablaje Catastral 13615, Colonia Chuburna Hidalgo Inn, 97203 Mérida, YUC, Mexico

Correspondence should be addressed to Eric ÁvilaVales, avila@uady.mx

Received 7 March 2012; Accepted 27 August 2012

Academic Editor: Alexander Timokha

Copyright (C) 2012 Noé Chan Chí et al. This is an open access article distributed under the Creative Commons Attribution License, which permits unrestricted use, distribution, and reproduction in any medium, provided the original work is properly cited.

This paper discussed a hepatitis B virus infection with delay, spatial diffusion, and standard incidence function. The local stability of equilibrium is obtained via characteristic equations. By using comparison arguments, it is proved that if the basic reproduction number is less than unity, the infection-free equilibrium is globally asymptotically stable. If the basic reproductive number is greater than unity, by means of an iteration technique, sufficiently conditions are obtained for the global asymptotic stability of the infected steady state. Numerical simulations are carried out to illustrate our findings.

\section{Introduction}

Human infection with hepatitis B virus (HBV) is a major global health problem. Between 300 and 400 million people are chronically infected worldwide. The virus is contracted through contact with blood or other fluids from the body, which could lead to develop viral persistence in the individual in the absence of strong antibody or some immune depression. Mathematical models have the potential to improve the understanding of the dynamics of this disease; one of the earliest models is referred to as the basic virus infection model, introduced by Nowak et al. [1]. They proposed a basic mathematical model for uninfected susceptible host cells (hepatocytes), $u$, infected host cells, $w$, and free virus particles, $v$, as follows:

$$
\begin{aligned}
& \dot{u}(t)=s-\mu u(t)-\beta u(t) v(t), \\
& \dot{w}(t)=\beta u(t) v(t)-a w(t), \\
& \dot{v}(t)=k w(t)-d v(t),
\end{aligned}
$$


where hepatocytes are produced at a rate $s$, uninfected cells die at rate $\mu$ and become and infected at rate $\beta u(t) v(t)$, infected hepatocytes are produced at rate $\beta u(t) v(t)$ and die at rate $a w(t)$. Free viruses are produced from infected cells at rate $k w(t)$ and are removed at rate $d v(t)$. It is assumed that all parameters are positive constants. Previous models assume that the infectious process is instantaneous; that is, in the very moment that the virus enters an uninfected cell, this one starts to produce virus particles; we know that this is not biologically reasonable. Thus, models with delays have been considered; in [2], the authors studied the following hepatitis B virus infection model with a time delay:

$$
\begin{aligned}
& \dot{x}(t)=\lambda-d x(t)-\frac{\beta x(t) v(t)}{x(t)+y(t)} \\
& \dot{y}(t)=\frac{\beta \mathrm{e}^{-m \tau} x(t-\tau) v(t-\tau)}{x(t-\tau)+y(t-\tau)}-a y(t) \\
& \dot{v}(t)=k y(t)-u v(t)
\end{aligned}
$$

The authors gave results about local and global stability of feasible equilibria.

For HBV infection, susceptible host cells and infected cells are hepatocytes and cannot move under normal conditions, but viruses move freely in liver [3]; therefore, the authors introduce an HBV model with diffusion and delay. $\mathrm{Xu}$ and Ma [4] considered also a diffusion model with delay but instead of bilinear response of the infection rate, they considered saturation response.

In this work motivated by the work of $\mathrm{Xu}$ and $\mathrm{Ma}$, we study the following model:

$$
\begin{aligned}
& \frac{\partial u}{\partial t}=L-d u(x, t)-\frac{\beta u(x, t) v(x, t)}{u(x, t)+w(x, t)} \\
& \frac{\partial w}{\partial t}=\frac{\beta \mathrm{e}^{-m \tau} u(x, t-\tau) v(x, t-\tau)}{u(x, t-\tau)+w(x, t-\tau)}-a w(x, t), \\
& \frac{\partial v}{\partial t}=D \Delta v+k w(x, t)-p v(x, t)
\end{aligned}
$$

for $t>0, x \in \Omega$, with homogeneous Neumann boundary conditions

$$
\frac{\partial v}{\partial \eta}=0
$$

and initial conditions

$$
\begin{gathered}
u(x, \theta)=\phi_{1}(x, \theta) \geq 0, \quad w(x, \theta)=\phi_{2}(x, \theta) \geq 0, \\
v(x, \theta)=\phi_{3}(x, \theta) \geq 0, \quad \theta \in[-\tau, 0], \quad x \in \bar{\Omega} .
\end{gathered}
$$

In the previous problem $\Omega$ is a bounded domain in $\mathbb{R}^{n}$ with smooth boundary $\partial \Omega, \partial / \partial \eta$ denotes the outward normal derivative on $\partial \Omega$.

This paper is ordered as follows. In the next section we present a result about the existence, uniqueness, and positivity. In Section 3 we discuss the local stability of each 
of the feasible equilibria of system (1.3), by analyzing the corresponding characteristic equations. In Section 4, by using comparison arguments and an iterative technique, we establish sufficient conditions for the global stability of the equilibria of system (1.3). In Section 5 numerical simulations are carried out to illustrate our principal results and we compare the effect of the diffusion and the delay on the system (1.3).

\section{Preliminaries}

Consider problem (1.3)-(1.5) and the following definitions.

Definition 2.1. A pair of functions $\tilde{U}=(\widetilde{u}, \widetilde{w}, \widetilde{v}), \widehat{U}=(\widehat{u}, \widehat{w}, \widehat{v})$ in $C([0, \infty) \times \bar{\Omega}) \cap C^{(1,2)}((0, \infty) \times$ $\Omega$ ) are called coupled upper and lower solutions to system (1.3)-(1.5) if $\widehat{u} \leq \tilde{u}, \widehat{w} \leq \tilde{w}, \widehat{v} \leq \tilde{v}$ in $\bar{\Omega} \times[-\tau, \infty)$ and the following differential inequalities hold:

$$
\begin{aligned}
& \frac{\partial \tilde{u}}{\partial t} \geq L-d \tilde{u}(x, t)-\frac{\beta \tilde{u}(x, t) \widehat{v}(x, t)}{\widetilde{u}(x, t)+\widehat{w}(x, t)}, \\
& \frac{\partial \tilde{w}}{\partial t} \geq \frac{\beta \mathrm{e}^{-m \tau} \tilde{u}(x, t-\tau) \tilde{v}(x, t-\tau)}{\widetilde{u}(x, t-\tau)+\tilde{w}(x, t-\tau)}-a \tilde{w}(x, t), \\
& \frac{\partial \widetilde{v}}{\partial t} \geq D \Delta \widetilde{v}+k \tilde{w}(x, t)-p \widetilde{v}(x, t), \\
& \frac{\partial \widehat{u}}{\partial t} \leq L-d \tilde{u}(x, t)-\frac{\beta \tilde{u}(x, t) \widehat{v}(x, t)}{\widetilde{u}(x, t)+\widehat{w}(x, t)}, \\
& \frac{\partial \widehat{w}}{\partial t} \leq \frac{\beta \mathrm{e}^{-m \tau} \widehat{u}(x, t-\tau) \widehat{v}(x, t-\tau)}{\widehat{u}(x, t-\tau)+\widehat{w}(x, t-\tau)}-a \widehat{w}(x, t), \\
& \frac{\partial \widehat{v}}{\partial t} \leq D \Delta \widehat{v}+k \widehat{w}(x, t)-p \widehat{v}(x, t),
\end{aligned}
$$

for $(x, t) \in \Omega \times(0, \infty)$, and

$$
\begin{gathered}
\frac{\partial \widehat{u}}{\partial \eta} \leq 0 \leq \frac{\partial \widetilde{u}}{\partial \eta}, \quad \frac{\partial \widehat{w}}{\partial \eta} \leq 0 \leq \frac{\partial \tilde{w}}{\partial \eta}, \\
\frac{\partial \widehat{v}}{\partial \eta} \leq 0 \leq \frac{\partial \widetilde{v}}{\partial \eta} \quad(x, t) \in \partial \Omega \times(0, \infty), \\
\widehat{u}(x, t) \leq \phi_{1}(x, t) \leq \tilde{u}(x, t), \quad \widehat{w}(x, t) \leq \phi_{2}(x, t) \leq \tilde{w}(x, t), \\
\widehat{v}(x, t) \leq \phi_{3}(x, t) \leq \tilde{v}(x, t), \quad(x, t) \in \bar{\Omega} \times[-\tau, 0] .
\end{gathered}
$$

The following lemma then follows from Theorem 3.4 developed by Redlinger [5].

Lemma 2.2. Let $\tilde{U}$ and $\widehat{U}$ be a pair of coupled upper and lower solutions for problem (1.3)-(1.5) and suppose that the initial functions $\phi_{i}(i=1,2,3)$ are Hölder continuous in $[-\tau, 0] \times \Omega$. Then 
problem (1.3)-(1.5) has exactly one regular solution $U(x, t)=(u(x, t), w(x, t), v(x, t))$ satisfying $\widehat{U} \leq U \leq \tilde{U}$ in $\bar{\Omega} \times[-\tau, \infty)$.

It is not hard to see that $0=(0,0,0)$ and $K=\left(K_{1}, K_{2}, K_{3}\right)$ are a pair of coupled lowerupper solutions to problem (1.3)-(1.5), where

$$
\begin{aligned}
& K_{1}=\max \left\{\frac{L}{d^{\prime}} \sup _{-\tau \leq \theta \leq 0}\left\|\phi_{1}(\cdot, \theta)\right\|_{C(\bar{\Omega}, R)}\right\}, \\
& K_{2}=\max \left\{\frac{k \beta \mathrm{e}^{-m \tau} K_{1}}{a p}, \sup _{-\tau \leq \theta \leq 0}\left\|\phi_{2}(\cdot, \theta)\right\|_{C(\bar{\Omega}, R)}\right\}, \\
& K_{3}=\max \left\{\frac{k^{2} \beta \mathrm{e}^{-m \tau} K_{1}}{a p^{2}}, \sup _{-\tau \leq \theta \leq 0}\left\|\phi_{3}(\cdot, \theta)\right\|_{C(\bar{\Omega}, R)}\right\} .
\end{aligned}
$$

Hence, $0 \leq u(x, t) \leq K_{1}, 0 \leq w(x, t) \leq K_{2}, 0 \leq v(x, t) \leq K_{3}$ for $(x, t) \in \bar{\Omega} \times[-\tau, \infty)$, and also, by the maximum principle, if $\phi_{i}(x, 0) \not \equiv 0(i=1,2,3)$, we have $u(x, t)>0, w(x, t)>0, v(x, t)>0$ for all $t>0, x \in \bar{\Omega}$.

\section{Local Stability}

System (1.3) has the equilibrium $E_{1}(L / d, 0,0)$. Let $R_{0}=\beta k \mathrm{e}^{-m \tau} / a p>1$ then system (1.3) has a unique infected steady state $E_{2}\left(u^{*}(\tau), w^{*}(\tau), v^{*}(\tau)\right)$; the previous notation is because the equilibrium involves $\tau$ and we use this as the parameter for the stability analysis, where

$$
\begin{gathered}
u^{*}(\tau)=\frac{L \mathrm{e}^{-m \tau}}{d \mathrm{e}^{-m \tau}+a\left(R_{0}-1\right)}, \quad w^{*}(\tau)=\frac{L \mathrm{e}^{-m \tau}\left(R_{0}-1\right)}{d \mathrm{e}^{-m \tau}+a\left(R_{0}-1\right)}, \\
v^{*}(\tau)=\frac{L k \mathrm{e}^{-m \tau}\left(R_{0}-1\right)}{p\left[d \mathrm{e}^{-m \tau}+a\left(R_{0}-1\right)\right]} .
\end{gathered}
$$

Let $0=\mu_{1}<\mu_{2}<\cdots$ be the eigenvalues of the operator $-\Delta$ on $\Omega$ with the homogeneous Neumann boundary conditions, and let $E\left(\mu_{i}\right)$ be the eigenspace corresponding to $\mu_{i}$ in $C^{1}(\Omega)$.

Let $\mathbb{X}=\left[C^{1}(\Omega)\right]^{3}$, let $\left\{\phi_{i j} ; j=1,2, \ldots, \operatorname{dim} E\left(\mu_{i}\right)\right\}$ be an orthonormal basis of $E\left(\mu_{i}\right)$, and let $\mathbb{X}_{i j}=\left\{c \phi_{i j} \mid c \in \mathbb{R}^{3}\right\}$, then

$$
\mathbb{X}=\bigoplus_{i=0}^{\infty} \mathbb{X}_{i}, \quad \mathbb{X}_{i}=\bigoplus_{j=1}^{\operatorname{dim} E\left(p_{i}\right)} \mathbb{X}_{i j}
$$


Let $\Phi=\operatorname{diag}(0,0, D), Z=Z(x, t)=(U(x, t), W(x, t), V(x, t)), \mathcal{L} Z=\Phi \Delta Z+J_{E^{*}} Z+J_{\tau E^{*}} Z(t-\tau)$, where

$$
\begin{aligned}
J_{E^{*}} & =\left(\begin{array}{ccc}
-d-\frac{\beta \bar{w} \bar{v}}{(\bar{u}+\bar{w})^{2}} & \frac{\beta \bar{u} \bar{v}}{(\bar{u}+\bar{w})^{2}} & -\frac{\beta \bar{u}}{\bar{u}+\bar{w}} \\
0 & -a & 0 \\
0 & k & -p
\end{array}\right), \\
J_{\tau} & =\left(\begin{array}{ccc}
0 & 0 & 0 \\
\frac{\beta \mathrm{e}^{-m \tau} \bar{v} \bar{w}}{(\bar{u}+\bar{w})^{2}} & -\frac{\beta \mathrm{e}^{-m \tau} \bar{u} \bar{v}}{(\bar{u}+\bar{w})^{2}} & \frac{\beta \mathrm{e}^{-m \tau} \bar{u}}{\bar{u}+\bar{w}} \\
0 & 0 & 0
\end{array}\right),
\end{aligned}
$$

and $E^{*}(\bar{u}, \bar{w}, \bar{v})$ represents any feasible steady state of the system (1.3). The linearization of system (1.3) at $E^{*}$ is of the form $Z_{t}=\mathcal{L Z}$. For each $i \geq 1, \mathbb{X}_{i}$ is invariant under the operator $\mathcal{L}$, and $\mathcal{\lambda}$ is an eigenvalue of the matrix $-\mu_{i} \Phi+J_{E^{*}}+J_{\tau E^{*}}$ for some $i \geq 1$, then, there is an eigenvector in $\mathbb{X}_{i}$.

The characteristic equation on the equilibrium $E_{1}$ is

$$
(\lambda+d)\left(\lambda^{2}+a_{1} \lambda+a_{0}+b_{0}(\tau) \mathrm{e}^{-\lambda \tau}\right)=0
$$

where

$$
a_{0}=a\left(p+\mu_{I} D\right), \quad a_{1}=a+p+\mu_{i} D, \quad b_{0}(\tau)=-k \beta \mathrm{e}^{-m \tau} .
$$

The characteristic equation has the negative root $\lambda=-d$. All other roots of (3.4) are given by the transcendental equation

$$
\lambda^{2}+a_{1} \lambda+a_{0}+b_{0}(\tau) \mathrm{e}^{-\lambda \tau}=0
$$

Let

$$
f(\lambda)=\lambda^{2}+a_{1} \lambda+a_{0}+b_{0}(\tau) \mathrm{e}^{-\lambda \tau},
$$

if $R_{0}>1$, note that for $\lambda$ real and $i=1$ (in this case $\mu_{1}=0$ ),

$$
f(0)=a_{0}+b_{0}=a p-k \beta \mathrm{e}^{-m \tau}<0, \quad \lim _{\lambda \rightarrow \infty} f(\lambda)=+\infty
$$

Hence, (3.7) has a positive root. Therefore, there is a characteristic root $\lambda$ with positive real part in the spectrum of $\mathcal{L}$. Accordingly, if $R_{0}>1$, the disease-free steady state $E_{1}(\lambda / d, 0,0)$ is unstable.

If $R_{0}<1$, when $\tau=0$ the coefficients of (3.7) are $a_{1}$ and $a_{0}+b_{0}(0)$, and under the hypothesis $R_{0}<1$ the coefficients are positive and according to the criterion of RouthHurwitz, the equilibrium $E_{1}(\lambda / d, 0,0)$ is locally asymptotically stable. 
For $\tau>0$ if $i \omega(\omega>0)$ is a solution of (3.6), separating in real and imaginary parts, we obtain

$$
\begin{gathered}
-\omega^{2}+a_{0}=-b_{0}(\tau) \cos (\omega \tau), \\
a_{1} \omega=b_{0}(\tau) \sin (\omega \tau) .
\end{gathered}
$$

Squaring and adding the above equations and taking $z=\omega^{2}$ we obtain

$$
z^{2}+\left(a_{1}^{2}-2 a_{0}\right) z+a_{0}^{2}-b_{0}(\tau)^{2}=0
$$

where

$$
\begin{gathered}
a_{1}^{2}-2 a_{0}=a^{2}+\left(p+\mu_{i} D\right)^{2}>0, \\
a_{0}^{2}-b_{0}^{2}(\tau)=\left(a\left(p+\mu_{i} D\right)-k \beta \mathrm{e}^{-m \tau}\right)\left(a\left(p+\mu_{i} D\right)+k \beta \mathrm{e}^{-m \tau}\right)>0,
\end{gathered}
$$

the last inequality is true because $R_{0}<1$. Therefore there is no positive root $z=\omega^{2}$ of (3.10). In conclusion if $R_{0}<1$ the equilibrium $E_{1}(\lambda / d, 0,0)$ is locally asymptotically stable.

The characteristic equation of system (1.3) at the endemic equilibrium $E_{2}\left(u^{*}, w^{*}, v^{*}\right)$ is of the form

$$
\lambda^{3}+a_{2}(\tau) \lambda^{2}+a_{1} \lambda+a_{0}(\tau)+\left(b_{2}(\tau) \lambda^{2}+b_{1}(\tau) \lambda+b_{0}(\tau)\right) \mathrm{e}^{-\lambda \tau}=0,
$$

where

$$
\begin{gathered}
a_{2}(\tau)=a+d+p+\mu_{i} D+\frac{\beta v^{*} w^{*}}{\left(u^{*}+w^{*}\right)^{2}}, \\
a_{1}=a\left(p+\mu_{i} D\right)+\left(a+p+\mu_{i} D\right) d, \\
a_{0}(\tau)=a\left(p+\mu_{i} D\right)\left[d+\frac{\beta w^{*} v^{*}}{\left(u^{*}+w^{*}\right)^{2}}\right], \\
b_{2}(\tau)=\frac{\beta \mathrm{e}^{-m \tau} u^{*} v^{*}}{\left(u^{*}+w^{*}\right)^{*}}, \\
b_{1}(\tau)=\left(d+p+\mu_{i} D\right) \frac{\beta \mathrm{e}^{-m \tau} u^{*} w^{*}}{\left(u^{*}+w^{*}\right)^{2}}-k \frac{\beta \mathrm{e}^{-m \tau} u^{*}}{u^{*}+w^{*}}, \\
b_{0}(\tau)=d\left(p+\mu_{i} D\right) \frac{\beta \mathrm{e}^{-m \tau} u^{*} v^{*}}{\left(u^{*}+w^{*}\right)^{2}}-d k \frac{\beta \mathrm{e}^{-m \tau} u^{*}}{u^{*}+w^{*}},
\end{gathered}
$$

when $\tau=0$ becomes

$$
\lambda^{3}+\left(a_{2}(0)+b_{2}(0)\right) \lambda^{2}+\left(a_{1}+b_{1}(0)\right) \lambda+\left(a_{0}(0)+b_{0}(0)\right)=0 .
$$


Note that $a_{2}(0)+b_{2}(0)>0$; adding $a_{0}(0)+b_{0}(0)$ and replacing $u^{*}(0)$ and $w^{*}(0)$ we obtain

$$
\begin{aligned}
a_{0}(0)+ & b_{0}(0)=a\left(p+\mu_{i} D\right) \frac{\beta w^{*}(0) v^{*}(0)}{\left(u^{*}(0)+w^{*}(0)\right)^{2}}+d\left(p+\mu_{i} D\right) \frac{\beta u^{*}(0) v^{*}(0)}{\left(u^{*}(0)+w^{*}(0)\right)^{2}}>0, \\
\left(a_{2}(0)+b_{2}(0)\right)\left(a_{1}+b_{1}(0)\right)-a_{0}(0)+b_{0}(0) & \left(d\left(a+p+\mu_{i} D\right)+\left(u^{*}(0)+w^{*}(0)\right) \frac{\left(p+\mu_{i} D\right) \beta v^{*}(0)}{\left(u^{*}(0)+w^{*}(0)\right)^{2}}\right) \\
= & \left(a+d+p+\mu_{i} D+\left(u^{*}(0)+w^{*}(0)\right) \frac{\beta v^{*}}{\left(u^{*}(0)+w^{*}(0)\right)^{2}}\right) \\
& +\frac{\lambda \beta v^{*}(0)}{\left(u^{*}(0)+w^{*}(0)\right)^{2}}\left(a+d+\left(u^{*}(0)+w^{*}(0)\right) \frac{\beta v^{*}(0)}{\left(u^{*}(0)+w^{*}(0)\right)^{2}}\right)>0 .
\end{aligned}
$$

By the Routh-Hurwitz criteria, all roots have negative real parts if $R_{0}>1$.

For the case $\tau>0$ we look for solutions $\lambda=i \omega(\omega>0)$ for (3.12), separating real and imaginary parts, it follows that

$$
\begin{gathered}
\omega^{3}-a_{1} \omega=\left(b_{2}(\tau) \omega^{2}-b_{0}(\tau)\right) \sin (\omega \tau)+b_{1}(\tau) \omega \cos (\omega \tau), \\
a_{2}(\tau) \omega^{2}-a_{0}(\tau)=-\left(b_{2}(\tau) \omega^{2}-b_{0}(\tau)\right) \cos (\omega \tau)+b_{1}(\tau) \omega \sin (\omega \tau) .
\end{gathered}
$$

Squaring and adding the two equations, we derive that

$$
\omega^{6}+C_{1} \omega^{4}+C_{2} \omega^{2}+C_{3}=0
$$

where

$$
\begin{aligned}
C_{1}= & \left(p+\mu_{i} D\right)^{2}+\left(d+\frac{\beta w^{*}(\tau) v^{*}(\tau)}{\left(u^{*}(\tau)+w^{*}(\tau)\right)^{2}}\right)^{2}+\frac{a u^{*}(\tau)}{u^{*}(\tau)+w^{*}(\tau)}\left(a+\frac{\beta \mathrm{e}^{-m \tau} u^{*}(\tau) v^{*}(\tau)}{\left(u^{*}(\tau)+w^{*}(\tau)\right)^{2}}\right)>0 \\
C_{2}= & a^{2}\left(d^{2}+\left(p+\mu_{i} D\right)^{2}\right) \frac{w^{*}(\tau)\left(u^{*}(\tau)+2 w^{*}(\tau)\right)}{\left(u^{*}(\tau)+w^{*}(\tau)\right)^{2}} \\
& +\frac{a^{2} \beta w^{*}(\tau) v^{*}(\tau)}{\left(u^{*}(\tau)+w^{*}(\tau)\right)^{2}}\left(2 d+\frac{\beta w^{*}(\tau) v^{*}(\tau)}{\left(u^{*}(\tau)+w^{*}(\tau)\right)^{2}}\right)+\left(p+\mu_{i} D\right)\left(d+\frac{\beta w^{*}(\tau)+v^{*}(\tau)}{\left(u^{*}(\tau)+w^{*}(\tau)\right)^{2}}\right)>0 \\
C_{3}= & \frac{a\left(p+\mu_{i} D\right)^{2} \beta v^{*}(\tau)\left(d \mathrm{e}^{-m \tau} u^{*}(\tau)+a w^{*}(\tau)\right)}{\left(u^{*}(\tau)+w^{*}(\tau)\right)^{3}}\left(d\left(2 x^{*}(\tau)+w^{*}(\tau)+\frac{\beta w^{*}(\tau) v^{*}(\tau)}{u^{*}(\tau)+w^{*}(\tau)}\right)\right)>0
\end{aligned}
$$

implying that (3.17) has no positive roots $z=\omega^{2}$. 
Theorem 3.1. If $R_{0}<1$ the disease-free equilibrium is locally asymptotically stable; if $R_{0}>1$ it is unstable and the endemic equilibrium exists and it is locally asymptotically stable.

\section{Global Stability}

We will discuss in this section the global stability of the infected steady state and the diseasefree equilibrium. The technique of proof is to use comparison arguments and to successively modify the coupled lower-upper solutions pairs.

Consider the following delay system:

$$
\begin{aligned}
& \dot{u}_{1}(t)=\frac{a_{1} \beta \mathrm{e}^{-m \tau} u_{2}(t-\tau)}{a_{1}+u_{1}(t-\tau)}-a u_{1}(t), \\
& \dot{u}_{2}(t)=k u_{1}(t)-p u_{2}(t)
\end{aligned}
$$

with initial conditions

$$
u_{i}(s)=\phi_{i}(s) \geq 0, \quad s \in[-\tau, 0), \phi_{i}(0)>0, \phi_{i} \in C\left([-\tau, 0], \mathbb{R}_{+}\right) .
$$

System (4.1) always have the trivial equilibrium $A^{0}(0,0)$. If $k \beta \mathrm{e}^{-m \tau}>a p$, then system (4.1) has a unique positive equilibrium $A^{*}\left(u_{1}^{*}, u_{2}^{*}\right)$ where

$$
u_{1}^{*}=\frac{a_{1}\left(k \beta \mathrm{e}^{-m \tau}-a p\right)}{a p}, \quad u_{2}^{*}=\frac{a_{1} k\left(k \beta \mathrm{e}^{-m \tau}-a p\right)}{a p^{2}}
$$

and according to [2], for system (4.1), one has the following.

Lemma 4.1. If $k \beta \mathrm{e}^{-m \tau}>$ ap, then the positive equilibrium $A^{*}\left(u_{1}^{*}, u_{2}^{*}\right)$ is globally stable.

If $k \beta \mathrm{e}^{-m \tau}<$ ap, then the equilibrium $A^{0}(0,0)$ is globally stable.

Now we stablish and prove our result about global stability.

Theorem 4.2. Let $(u(x, t), w(x, t), v(x, t))$ be a solution to problem (1.3)-(1.5), let $\phi_{i}(x, 0) \not \equiv 0(i=$ $1,2,3)$. If $R_{0}>1$ and

(H1) $d p \mathrm{e}^{-m \tau}>k \beta \mathrm{e}^{-m \tau}-a p$,

then

$$
\lim _{t \rightarrow \infty}(u(x, t), w(x, t), v(x, t))=\left(u^{*}, w^{*}, v^{*}\right) \quad \text { uniformly for } x \in \Omega
$$

that is, the infected steady state $E^{*}$ is globally asymptotically stable. 
Proof. Let $(u(x, t), w(x, t), v(x, t))$ be a solution to problem (1.3)-(1.5), let $\phi_{i} \not \equiv 0(i=1,2,3)$. We have $u(x, t)>0, w(x, t)>0$, and $v(x, t)>0$ for all $x \in \bar{\Omega}, t>0$. Denote

$$
\begin{array}{ll}
\bar{u}=\limsup _{t \rightarrow \infty} \max _{x \in \bar{\Omega}} u(x, t), \quad \underline{u}=\liminf _{t \rightarrow \infty} \min _{x \in \bar{\Omega}} u(x, t), \\
\bar{w}=\limsup _{t \rightarrow \infty} \max _{x \in \bar{\Omega}} w(x, t), \quad \underline{w}=\liminf _{t \rightarrow \infty} \min _{x \in \bar{\Omega}} w(x, t), \\
\bar{v}=\limsup _{t \rightarrow \infty} \max _{x \in \bar{\Omega}} v(x, t), \quad \underline{v}=\liminf _{t \rightarrow \infty} \min _{x \in \bar{\Omega}} v(x, t) .
\end{array}
$$

First we look for upper solutions for the system (1.3). Let $\left(\bar{u}^{1}(x, t), \bar{w}^{1}(x, t), \bar{v}^{1}(x, t)\right)$ be a solution for the following problem:

$$
\begin{gathered}
\frac{\partial \bar{u}^{(1)}}{\partial t}=L-d \bar{u}^{(1)}(x, t), \quad t>0, x \in \Omega, \\
\frac{\partial \bar{w}^{(1)}}{\partial t}=\frac{\beta \mathrm{e}^{-m \tau} \bar{u}^{(1)}(x, t-\tau) \bar{v}^{(1)}(x, t-\tau)}{\bar{u}^{(1)}(x, t-\tau)+\bar{w}^{(1)}(x, t-\tau)}-a \bar{w}^{(1)}(x, t), \quad t>0, x \in \Omega, \\
\frac{\partial \bar{v}^{(1)}}{\partial t}=D \Delta \bar{v}^{(1)}(x, t)+k \bar{w}^{(1)}(x, t)-p \bar{v}^{(1)}(x, t), \quad t>0, x \in \Omega, \\
\frac{\partial \bar{u}^{(1)}(x, t)}{\partial t}=\frac{\partial \bar{w}^{(1)}(x, t)}{\partial t}=\frac{\partial \bar{v}^{(1)}(x, t)}{\partial t}=0, \quad t>0, x \in \partial \Omega, \\
\bar{u}^{(1)}(x, t)=u(x, t), \quad \bar{v}^{(1)}(x, t)=v(x, t) \\
\bar{w}^{(1)}(x, t)=w(x, t), \quad t \in[-\tau, 0], x \in \bar{\Omega} .
\end{gathered}
$$

We note that the solution of this system is an upper solution of system (1.3)-(1.5). For $t>0$, $x \in \bar{\Omega}$ we have

$$
0 \leq u(x, t) \leq \bar{u}^{(1)}(x, t), \quad 0 \leq w(x, t) \leq \bar{w}^{(1)}(x, t), \quad 0 \leq v(x, t) \leq \bar{v}^{(1)}(x, t) .
$$

From the first equation of (4.6)

$$
\lim _{t \rightarrow \infty} \bar{u}^{(1)}(x, t)=\frac{L}{d}=M_{1}^{u}
$$

Hence, by comparison, for all $\epsilon>0$ sufficiently small, there exists $t_{1}>0$ such that if $t>t_{1}$

$$
\max _{x \in \bar{\Omega}} u^{(1)}(x, t) \leq M_{1}^{u}+\epsilon,
$$

since $\epsilon$ is arbitrary and sufficiently small we can conclude that

$$
\bar{u}=\limsup _{t \rightarrow \infty} \max _{x \in \bar{\Omega}} u(x, t) \leq M_{1}^{u} .
$$


Now consider the problem related with the second and third equations of (4.6)

$$
\begin{gathered}
\frac{\partial \omega_{2}^{(1)}}{\partial t}=\frac{\beta \mathrm{e}^{-m \tau}\left(M_{1}^{u}+\epsilon\right) \omega_{3}^{(1)}(x, t-\tau)}{M_{1}^{u}+\epsilon+\omega_{2}^{(1)}(x, t-\tau)}-a \omega_{2}^{(1)}(x, t), \quad t>t_{1}, x \in \Omega, \\
\frac{\partial \omega_{3}^{(1)}}{\partial t}=D \Delta \omega_{3}^{(1)}(x, t)+k \omega_{2}^{(1)}(x, t)-p \omega_{3}^{(1)}, \quad t>t_{1}, x \in \Omega, \\
\frac{\partial \omega_{2}^{(1)}}{\partial \eta}=\frac{\partial \omega_{3}^{(1)}}{\partial \eta}=0, \quad t>t_{1}, x \in \partial \Omega, \\
\omega_{2}^{(1)}(x, t)=w(x, t), \quad \omega_{3}^{(1)}(x, t)=v(x, t), \quad t \in\left[-\tau, t_{1}\right], x \in \bar{\Omega} .
\end{gathered}
$$

Consider the solution for

$$
\begin{gathered}
\dot{u}_{2}=\frac{\beta \mathrm{e}^{-m \tau}\left(M_{1}^{u}+\epsilon\right) u_{3}(t-\tau)}{M_{1}^{u}+\epsilon+u_{2}(t-\tau)}-a u_{2}, \quad t>t_{1}, \\
\dot{u}_{3}=k u_{2}-p u_{3}, \quad t>t_{1}, \\
u_{2}(t)=\max _{x \in \bar{\Omega}} w(x, t), \quad u_{3}(t)=\max _{x \in \bar{\Omega}} v(x, t), \quad t \in\left[-\tau, t_{1}\right] .
\end{gathered}
$$

Note that $\left(u_{1}(t), u_{2}(t)\right)$ is an upper solution for system (4.11), and using the assumption that $R_{0}>1$, by Lemma 4.1, it follows from (4.12) that

$$
\begin{aligned}
& \lim _{t \rightarrow \infty} u_{2}(t)=\frac{\left(k \beta \mathrm{e}^{-m \tau}-a p\right)\left(M_{1}^{u}+\epsilon\right)}{a p}, \\
& \lim _{t \rightarrow \infty} u_{3}(t)=\frac{k\left(k \beta \mathrm{e}^{-m \tau}-a p\right)\left(M_{1}^{u}+\epsilon\right)}{a p^{2}} .
\end{aligned}
$$

Hence, for all $\epsilon>0$ sufficiently small, by comparison there exists a $t_{2}>t_{1}$ such that if $t>t_{2}$

$$
\max _{x \in \bar{\Omega}} \bar{w}^{(1)}(x, t)<M_{1}^{w}+\epsilon, \quad \max _{x \in \bar{\Omega}} \bar{v}^{(1)}(x, t)<M_{1}^{v}+\epsilon,
$$

where

$$
M_{1}^{w}=\frac{\left(k \beta \mathrm{e}^{-m \tau}-a p\right) M_{1}^{u}}{a p}, \quad M_{1}^{v}=\frac{k\left(k \beta \mathrm{e}^{-m \tau}-a p\right) M_{1}^{u}}{a p^{2}} .
$$

Since $\epsilon>0$ is arbitrary and sufficiently small, we conclude that

$$
\begin{aligned}
& \bar{w}=\limsup _{t \rightarrow \infty} \max _{x \in \bar{\Omega}} w(x, t) \leq M_{1}^{w}, \\
& \bar{v}=\limsup _{t \rightarrow \infty} \max _{x \in \bar{\Omega}} v(x, t) \leq M_{1}^{v} .
\end{aligned}
$$


Now for lower solutions, let $\left(\underline{u}^{(1)}(x, t), \underline{w}^{(1)}(x, t), \underline{v}^{(1)}(x, t)\right)$ be the solution for the following problem:

$$
\begin{gathered}
\frac{\partial \underline{u}^{(1)}}{\partial t}=L-d \underline{u}^{(1)}(x, t)-\frac{\beta \underline{u}^{(1)}(x, t) \bar{v}^{(1)}(x, t)}{\underline{u}^{(1)}(x, t)+\bar{w}^{(1)}(x, t)}, \quad t>t_{2}, \quad x \in \Omega, \\
\frac{\partial \underline{w}^{(1)}}{\partial t}=\frac{\beta \mathrm{e}^{-m \tau} \underline{u}^{(1)}(x, t) \underline{v}^{(1)}(x, t)}{\underline{u}^{(1)}(x, t)+\underline{w}^{(1)}(x, t)}-a \underline{w}^{(1)}(x, t), \quad t>t_{2}, \quad x \in \Omega, \\
\frac{\partial \underline{v}^{(1)}}{\partial t}=D \Delta \underline{v}^{(1)}(x, t)+k \underline{w}^{(1)}(x, t)-p \underline{v}^{(1)}(x, t), \quad t>t_{2}, x \in \Omega, \\
\quad \frac{\partial \underline{u}^{(1)}}{\partial \eta}=\frac{\partial \underline{w}^{(1)}}{\partial \eta}=\frac{\partial \underline{v}^{(1)}}{\partial \eta}=0, \quad t>t_{2}, x \in \partial \Omega, \\
\underline{u}^{(1)}(x, t)=\frac{1}{2} u(x, t), \quad \underline{w}^{(1)}(x, t)=\frac{1}{2} w(x, t), \\
\underline{v}^{(1)}(x, t)=\frac{1}{2} v(x, t), \quad t \in\left[-\tau, t_{2}\right], x \in \bar{\Omega} .
\end{gathered}
$$

Note that the solution of (4.17) is a lower solution to (1.3)-(1.5). For all $\epsilon>0$ sufficiently small, from the first equation of (4.17) and (4.16) it follows

$$
\frac{\partial \underline{u}^{(1)}}{\partial t} \geq L-d \underline{u}^{(1)}(x, t)-\beta\left(M_{1}^{v}+\epsilon\right), \quad t>t_{2}, x \in \Omega
$$

By comparing the above equation with the following problem:

$$
\begin{aligned}
& \frac{\partial \omega_{1}^{(1)}}{\partial t}=L-d \omega_{1}^{(1)}(x, t)-\beta\left(M_{1}^{v}+\epsilon\right), \quad t>t_{2}, x \in \Omega, \\
& \frac{\partial \omega_{1}}{\omega \eta}=0, \quad t>t_{2}, \quad x \in \partial \Omega, \quad \omega_{1}\left(x, t_{2}\right)=\frac{1}{2} u\left(x, t_{2}\right), \quad x \in \bar{\Omega},
\end{aligned}
$$

we obtain

$$
\lim _{t \rightarrow \infty} \omega_{1}(x, t)=\frac{L-\beta\left(M_{1}^{v}+\epsilon\right)}{d},
$$

so $\underline{u}^{(1)}(x, t) \geq \omega_{1}(x, t), t>t_{2}$, and $x \in \Omega$. Hence, for all $\epsilon>0$ sufficiently small, there is a $t_{3}>t_{2}$ such that if $t>t_{3}$,

$$
\min _{x \in \bar{\Omega}} \underline{u}^{(1)}(x, t) \geq N_{1}^{u}-\epsilon
$$


where

$$
N_{1}^{u}=\frac{L-\beta M_{1}^{v}}{d}
$$

Since $\epsilon>0$ is arbitrary sufficiently small, by comparison we conclude that

$$
\underline{u}=\liminf _{t \rightarrow \infty} \min _{x \in \bar{\Omega}} u(x, t) \geq N_{1}^{u}=\frac{L-\beta M_{1}^{v}}{d} .
$$

Now consider the following problem related with the second and third equations of (4.17):

$$
\begin{gathered}
\frac{\partial \omega_{2}}{\partial t}=\frac{\beta \mathrm{e}^{-m \tau}\left(N_{1}^{u}-\epsilon\right) \omega_{3}(x, t-\tau)}{N_{1}^{u}-\epsilon+\omega_{2}(x, t-\tau)}-a \omega_{2}(x, t), \quad t>t_{3}, x \in \Omega, \\
\frac{\partial \omega_{3}}{\partial t}=D \Delta \omega_{3}(x, t)+k \omega_{2}(x, t)-p \omega_{3}(x, t), \quad t>t_{3}, x \in \Omega, \\
\frac{\partial \omega_{2}}{\partial \eta}=\frac{\partial \omega_{3}}{\partial \eta}=0, \quad t>t_{3}, x \in \partial \Omega, \\
\omega_{2}(x, t)=\frac{1}{2} w(x, t), \quad \omega_{3}(x, t)=\frac{1}{2} v(x, t), \quad t \in\left[-\tau, t_{3}\right], x \in \bar{\Omega} .
\end{gathered}
$$

Now let us consider the solution for the problem

$$
\begin{gathered}
\dot{u}_{2}(t)=\frac{\beta \mathrm{e}^{-m \tau}\left(N_{1}^{u}-\epsilon\right) u_{3}(t-\tau)}{N_{1}^{u}-\epsilon+u_{2}(t-\tau)}-a u_{2}(t), \quad t>t_{3}, \\
\dot{u}_{3}(t)=k u_{2}(t)-p u_{3}(t), \quad t>t_{3}, \\
u_{2}(t)=\frac{1}{2} \min _{x \in \bar{\Omega}} w(x, t), \quad u_{3}(t)=\frac{1}{2} \min _{x \in \bar{\Omega}} v(x, t), \quad t \in\left[-\tau, t_{3}\right], t>t_{3},
\end{gathered}
$$

and according to Lemma 4.1

$$
\begin{aligned}
& \lim _{t \rightarrow \infty} u_{2}(t)=\frac{\left(k \beta \mathrm{e}^{-m \tau}-a p\right)\left(N_{1}^{u}-\epsilon\right)}{a p}, \\
& \lim _{t \rightarrow \infty} u_{3}(t)=\frac{k\left(k \beta \mathrm{e}^{-m \tau}-a p\right)\left(N_{1}^{u}-\epsilon\right)}{a p^{2}} .
\end{aligned}
$$

Hence, for all $\epsilon>0$ sufficiently small, by comparison there exists a $t_{4}>t_{3}$ such that if $t>t_{4}$

$$
\min _{x \in \bar{\Omega}} \underline{w}^{(1)}(x, t)>N_{1}^{w}-\epsilon, \quad \min _{x \in \bar{\Omega}} \underline{v}^{(1)}(x, t)>N_{1}^{v}-\epsilon,
$$


where

$$
N_{1}^{w}=\frac{\left(k \beta \mathrm{e}^{-m \tau}-a p\right) N_{1}^{u}}{a p}, \quad N_{1}^{v}=\frac{k\left(k \beta \mathrm{e}^{-m \tau}-a p\right) N_{1}^{u}}{a p^{2}} .
$$

Since $\epsilon>0$ is arbitrary and sufficiently small, we conclude that

$$
\begin{aligned}
& \underline{w}=\liminf _{t \rightarrow \infty} \min _{x \in \bar{\Omega}} w(x, t) \geq N_{1}^{w}, \\
& \underline{v}=\liminf _{t \rightarrow \infty} \min _{x \in \bar{\Omega}} v(x, t) \geq N_{1}^{v} .
\end{aligned}
$$

Now we look for the closest upper and lower solutions. Let $\left(\bar{u}^{(2)}, \bar{w}^{(2)}, \bar{v}^{(2)}\right)$ be a solution for the problem

$$
\begin{gathered}
\frac{\partial \bar{u}^{(2)}}{\partial t}=L-d \bar{u}^{(2)}(x, t)-\frac{\beta \bar{u}^{(2)}(x, t) \underline{v}^{(1)}(x, t)}{\bar{u}^{(1)}+\bar{w}^{(1)}(x, t)}, \quad t>t_{4}, x \in \Omega, \\
\frac{\partial \bar{w}^{(2)}}{\partial t}=\frac{\beta \mathrm{e}^{-m \tau} \bar{u}^{(2)}(x, t-\tau) \bar{v}^{(2)}(x, t)}{\bar{u}^{(2)}(x, t-\tau)+\bar{w}^{(2)}(x, t-\tau)}-a \bar{w}^{(2)}(x, t), \quad t>t_{4}, \quad x \in \Omega, \\
\frac{\partial \bar{v}^{(2)}}{\partial t}=D \Delta \bar{v}(x, t)+k \bar{w}^{(2)}(x, t)-p \bar{v}^{(2)}(x, t), \quad t>t_{4}, \quad x \in \Omega, \\
\frac{\partial \bar{u}^{(2)}}{\partial \eta}=\frac{\partial \bar{w}^{(2)}}{\partial \eta}=\frac{\partial \bar{v}^{(2)}}{\partial \eta}=0, \quad t>t_{4}, x \in \partial \Omega, \\
\bar{u}^{(2)}(x, t)=u(x, t), \quad \bar{w}^{(2)}(x, t)=w(x, t), \\
\bar{v}^{(2)}(x, t)=v(x, t), \quad t \in\left[-\tau, t_{4}\right], x \in \bar{\Omega} .
\end{gathered}
$$

For all $\epsilon>0$ sufficiently small it follows form the first equation of (4.30) and the inequalities (4.27) and (4.14) that

$$
\frac{\partial \bar{u}^{(2)}}{\partial t} \leq L-d \bar{u}^{(2)}(x, t)-\frac{\beta \bar{u}^{(2)}(x, t)\left(N_{1}^{v}-\epsilon\right)}{M_{1}^{u}+\epsilon+M_{1}^{w}+\epsilon}, \quad t>t_{4}, x \in \Omega .
$$

Let $\omega_{1}^{(2)}(x, t)$ be the solution for the following problem:

$$
\begin{gathered}
\frac{\partial \omega_{1}^{(2)}}{\partial t}=L-d \omega_{1}^{(2)}(x, t)-\frac{\beta \omega_{1}^{(2)}(x, t)\left(N_{1}^{v}-\epsilon\right)}{M_{1}^{u}+\epsilon+M_{1}^{w}+\epsilon}, \quad t>t_{4}, x \in \Omega, \\
\frac{\partial \omega_{1}^{(2)}}{\partial \eta}=0, \quad t>t_{4}, x \in \partial \Omega, \\
\omega_{1}^{(2)}\left(x, t_{4}\right)=u\left(x, t_{4}\right), \quad x \in \bar{\Omega},
\end{gathered}
$$


it follows that

$$
\lim _{t \rightarrow \infty} \omega_{1}^{(2)}(x, t)=\frac{L\left(M_{1}^{u}+M_{1}^{w}+2 \epsilon\right)}{d\left(M_{1}^{u}+M_{1}^{w}+2 \epsilon\right)+\beta\left(N_{1}^{v}-\epsilon\right)} .
$$

By comparison we have that $\bar{u}^{(2)} \leq \omega_{1}^{(2)}, t>t_{4}$, and $x \in \bar{\Omega}$. Hence, for all $\epsilon>0$ sufficiently small, by comparison, there is a $t_{5}>t_{4}$ such that if $t>t_{5}$

$$
\max _{x \in \bar{\Omega}} \bar{u}^{(2)}(x, t)=M_{2}^{u}+\epsilon,
$$

where

$$
M_{2}^{u}=\frac{L\left(M_{1}^{u}+M_{1}^{w}\right)}{d\left(M_{1}^{u}+M_{1}^{w}\right)+\beta\left(N_{1}^{v}\right)} .
$$

Since (4.34) is valid for $\epsilon>0$ arbitrary and sufficiently small, by comparison we conclude that

$$
\bar{u}=\limsup _{t \rightarrow \infty} \max _{x \in \bar{\Omega}} u(x, t) \leq M_{2}^{u} .
$$

Now consider the following problem related with the second and third equations of (4.30):

$$
\begin{gathered}
\frac{\partial \omega_{2}^{(2)}}{\partial t}=\frac{\beta \mathrm{e}^{-m \tau}\left(M_{2}^{u}+\epsilon\right) \omega_{3}^{(2)}(x, t)}{M_{2}^{u}+\omega_{2}^{(2)}(x, t-\tau)}-a \omega_{2}^{(2)}(x, t), \quad t>t_{5}, x \in \Omega, \\
\frac{\partial \omega_{3}^{(2)}}{\partial t}=D \Delta \omega_{3}^{(2)}(x, t)+k \omega_{2}^{(2)}(x, t)-p \omega_{3}^{(2)}(x, t), \quad t>t_{5}, x \in \Omega, \\
\quad \frac{\partial \omega_{2}^{(2)}}{\partial \eta}=\frac{\partial \omega_{3}^{(2)}}{\partial \eta}=0, \quad t>t_{5}, x \in \partial \Omega, \\
\omega_{2}^{(2)}(x, t)=w(x, t), \quad \omega_{3}^{(2)}(x, t)=v(x, t), \quad t \in\left[-\tau, t_{5}\right], x \in \bar{\Omega} .
\end{gathered}
$$

Let $\left(u_{2}(t), u_{3}(t)\right)$ be the positive solution to the following problem:

$$
\begin{aligned}
& \dot{u}_{2}(t)=\frac{\beta \mathrm{e}^{-m \tau}\left(M_{2}^{u}+\epsilon\right) u_{3}(t-\tau)}{M_{2}^{u}+\epsilon+u_{2}(t-\tau)}-a u_{2}(t), \quad t>t_{5}, \\
& \dot{u}_{3}(t)=k u_{2}(t)-p u_{3}(t), \quad t>t_{5}, \\
& u_{2}(t)=\max _{x \in \bar{\Omega}} w(x, t), \quad u_{3}(t)=\max _{x \in \bar{\Omega}} v(x, t), \quad t \in\left[-\tau, t_{5}\right] .
\end{aligned}
$$


Then by Lemma 4.1 and the previous system we have

$$
\begin{aligned}
\lim u_{2}(t) & =\frac{\left(k \beta \mathrm{e}^{-m \tau}-a p\right)\left(M_{2}^{u}+\epsilon\right)}{a p}, \\
\lim u_{3}(t) & =\frac{k\left(k \beta \mathrm{e}^{-m \tau}\right)\left(M_{2}^{u}+\epsilon\right)}{a p^{2}} .
\end{aligned}
$$

Hence for all $\epsilon>0$ sufficiently small, by comparison there is a $t_{6}>t_{5}$ such that if $t>t_{6}$

$$
\max _{x \in \bar{\Omega}} \bar{w}(x, t)<M_{2}^{w}+\epsilon, \quad \max _{x \in \bar{\Omega}} \bar{v}(x, t)<M_{2}^{v}+\epsilon,
$$

where

$$
\begin{aligned}
& M_{2}^{w}=\frac{\left(k \beta \mathrm{e}^{-m \tau}-a p\right) M_{2}^{u}}{a p}, \\
& M_{2}^{v}=\frac{k\left(k \beta \mathrm{e}^{-m \tau}\right) M_{2}^{u}}{a p^{2}} .
\end{aligned}
$$

Since $\epsilon>0$ is arbitrary and sufficiently small, we conclude that

$$
\bar{w}=\limsup _{t \rightarrow \infty} \max _{x \in \bar{\Omega}} w(x, t) \leq M_{2}^{w}, \quad \bar{v}=\limsup _{t \rightarrow \infty} \max _{x \in \bar{\Omega}} v(x, t) \leq M_{2}^{v} .
$$

Let $\left(\underline{u}^{(2)}, \underline{w}^{(2)}, \underline{v}^{(2)}\right)$ be a solution for the following problem:

$$
\begin{gathered}
\frac{\partial \underline{u}^{(2)}}{\partial t}=L-d \underline{u}^{(2)}(x, t)-\frac{\beta \underline{u}^{(2)}(x, t) \bar{v}^{(1)}(x, t)}{\underline{u}^{(1)}(x, t)+\underline{w}^{(1)}(x, t)}, \quad t>t_{6}, \quad x \in \Omega, \\
\frac{\partial \underline{w}^{(2)}}{\partial t}=\frac{\beta \mathrm{e}^{-m \tau} \underline{u}^{(2)}(x, t-\tau) \underline{v}^{(2)}(x, t-\tau)}{\underline{u}^{(2)}(x, t-\tau)+\underline{w}^{(2)}(x, t-\tau)}-a \underline{w}^{(2)}(x, t) \quad t>t_{6}, \quad x \in \Omega, \\
\frac{\partial \underline{v}}{\partial t}=D \Delta \underline{v}^{(2)}(x, t)+k \underline{w}^{(2)}-p \underline{v}^{(2)}(x, t) \quad t>t_{6}, x \in \Omega, \\
\underline{u}^{(2)}(x, t)=\frac{1}{2} u(x, t), \quad \underline{w}^{(2)}(x, t)=\frac{1}{2} w(x, t), \\
\underline{w}^{(2)}(x, t)=\frac{1}{2} v(x, t), \quad t \in\left[-\tau, t_{6}\right], x \in \bar{\Omega} .
\end{gathered}
$$

Then $\left(\underline{u}^{(2)}, \underline{w}^{(2)}, \underline{v}^{(2)}\right)$ and $\left(\bar{u}^{(2)}, \bar{w}^{(2)}, \bar{v}^{(2)}\right)$ are a pair of coupled lower and upper solutions to system (1.3)-(1.5). Hence we have that for $t \geq t_{6}, x \in \bar{\Omega}$

$$
\begin{gathered}
\underline{u}^{(2)}(x, t) \leq u(x, t) \leq \bar{u}^{(2)}(x, t), \quad \underline{w}^{(2)}(x, t) \leq w(x, t) \leq \bar{w}^{(2)}(x, t), \\
\underline{v}^{(2)}(x, t) \leq v(x, t) \leq \bar{v}^{(2)}(x, t) .
\end{gathered}
$$


For all $\epsilon>0$ sufficiently small, it follow from the first equation of (4.43), and the inequalities

$$
\frac{\partial \underline{u}^{(2)}}{\partial t} \geq L-d \underline{u}^{(2)}(x, t)-\frac{\beta \underline{u}^{(2)}(x, t)\left(M_{1}^{v}+\epsilon\right)}{N_{1}^{u}-\epsilon+N_{1}^{w}-\epsilon} .
$$

By comparison we have that $\underline{u}^{(2)}(x, t) \geq v_{1}^{(2)}(x, t), t>t_{6}$, and $x \in \bar{\Omega}$ where $v_{1}^{(2)}$ is the solution to problem

$$
\begin{gathered}
\frac{\partial v_{1}^{(2)}}{\partial t}=L-d v_{1}^{(2)}(x, t)-\frac{\beta v_{1}^{(2)}(x, t)\left(M_{1}^{v}+\epsilon\right)}{N_{1}^{u}-\epsilon+N_{1}^{w}-\epsilon}, \\
\frac{\partial v_{1}^{(2)}}{\partial \eta}=0, \quad t>t_{6}, x \in \partial \Omega,
\end{gathered}
$$

which has satisfies

$$
\lim _{t \rightarrow \infty} v_{1}^{(2)}(x, t)=\frac{L\left(N_{1}^{u}-\epsilon+N_{1}^{w}-\epsilon\right)}{d\left(N_{1}^{u}-\epsilon+N_{1}^{w}-\epsilon\right)+\beta\left(M_{1}^{v}+\epsilon\right)} .
$$

Hence for all $\epsilon>0$ sufficiently small, by comparison, there is a $t_{7}>t_{6}$ such that if $t>t_{7}$

$$
\min \underline{u}^{(2)}(x, t) \geq N_{2}^{u}-\epsilon,
$$

with

$$
N_{2}^{u}=\frac{L\left(N_{1}^{u}+N_{1}^{w}\right)}{d\left(N_{1}^{u}+N_{1}^{w}\right)+\beta M_{1}^{v}} .
$$

Since this holds true for arbitrary $\epsilon>0$ sufficiently small, by comparison we conclude that

$$
\underline{u}=\liminf _{t \rightarrow \infty} \min _{x \in \bar{\Omega}} u(x, t) \geq N_{2}^{u} .
$$

Now consider the following problem:

$$
\begin{gathered}
\frac{\partial v_{2}^{(2)}}{\partial t}=\frac{\beta \mathrm{e}^{-m \tau}\left(N_{2}^{u}-\epsilon\right) v_{3}^{(2)}(x, t-\tau)}{N_{2}^{u}-\epsilon+v_{2}^{(2)}(x, t-\tau)}-a v_{2}^{(2)}(x, t), \quad t>t_{7}, x \in \Omega, \\
\frac{\partial v_{3}^{(2)}}{\partial t}=D \Delta v_{3}^{(2)}(x, t)+k v_{2}^{(2)}-p v_{3}^{(2)}(x, t), \quad t>t_{7}, x \in \Omega, \\
\frac{\partial v_{2}^{(2)}}{\partial \eta}=\frac{\partial v_{3}^{(2)}}{\partial \eta}=0, \quad t>t_{7}, x \in \Omega, \\
v_{2}^{(2)}(x, t)=\frac{1}{2} w(x, t), \quad v_{3}^{(2)}(x, t)=\frac{1}{2} v(x, t), \quad t \in\left[-\tau, t_{7}\right], x \in \bar{\Omega} .
\end{gathered}
$$


Let $\left(u_{2}(t), u_{3}(t)\right)$ be the positive solution for the following problem:

$$
\begin{aligned}
& \dot{v}_{2}(t)=\frac{\beta \mathrm{e}^{-m \tau}\left(N_{2}^{u}-\epsilon\right) u_{2}(t-\tau)}{N_{2}^{u}-\epsilon+u_{2}(t-\tau)}-a u_{2}(t), \quad t>t_{7}, \\
& \dot{v}_{3}(t)=k v_{2}(t)-p v_{3}(t), \quad t>t_{7}, \\
& v_{2}(t)=\frac{1}{2} \min _{x \in \bar{\Omega}}, \quad v_{3}(t)=\frac{1}{2} \min _{x \in \bar{\Omega}} v(x, t), \quad t \in\left[-\tau, t_{7}\right] .
\end{aligned}
$$

By Lemma 4.1 it follows

$$
\lim _{t \rightarrow \infty} v_{2}(t)=\frac{\left(N_{2}^{u}-\epsilon\right)\left(k \beta \mathrm{e}^{-m \tau}-a p\right)}{a p}, \quad \lim _{t \rightarrow \infty} v_{3}(t)=\frac{k\left(N_{2}^{u}-\epsilon\right)\left(k \beta \mathrm{e}^{-m \tau}-a p\right)}{a p^{2}},
$$

hence, for all $\epsilon>0$ sufficiently small, by comparison there exists a $t_{8}>t_{7}$ such that if $t>t_{8}$,

$$
\min _{x \in \bar{\Omega}} \underline{w}^{(2)}(x, t)>N_{2}^{w}-\epsilon, \quad \min _{x \in \bar{\Omega}} \underline{v}^{(2)}(x, t)>N_{2}^{v}-\epsilon,
$$

where

$$
N_{2}^{w}=\frac{N_{2}^{u}\left(k \beta \mathrm{e}^{-m \tau}-a p\right)}{a p}, \quad N_{2}^{v}=\frac{k N_{2}^{u}\left(k \beta \mathrm{e}^{-m \tau}-a p\right)}{a p^{2}} .
$$

Since $\epsilon>0$ is arbitrary and sufficiently small, we conclude that

$$
\underline{w}=\liminf _{t \rightarrow \infty} \min _{x \in \bar{\Omega}} w(x, t) \geq N_{2}^{w}, \quad \underline{v}=\liminf _{t \rightarrow \infty} \min _{x \in \bar{\Omega}} v(x, t) \geq N_{2}^{v},
$$

continuing this process, we derive six sequences $M_{n}^{u}, M_{n}^{w}, M_{n}^{v}, N_{n}^{u}, N_{n}^{w}$, and $N_{n}^{v}(n=$ $1,2, \ldots)$ such that, for $n \geq 2$,

$$
\begin{aligned}
& M_{n}^{u}=\frac{L\left(M_{n-1}^{u}+M_{n-1}^{w}\right)}{d\left(M_{n-1}^{u}+M_{n-1}^{w}\right)+\beta N_{n-1}^{v}}, \\
& M_{n}^{w}=\frac{\left(k \beta \mathrm{e}^{-m \tau}-a p\right) M_{n}^{u}}{a p}, \\
& M_{n}^{v}=\frac{k\left(k \beta \mathrm{e}^{-m \tau}-a p\right) M_{n}^{u}}{a p^{2}}, \\
& N_{n}^{u}=\frac{L\left(N_{n-1}^{u}+N_{n-1}^{w}\right)}{d\left(N_{n-1}^{u}+N_{n-1}^{w}\right)+\beta M_{n}^{v}},
\end{aligned}
$$




$$
\begin{aligned}
& N_{n}^{w}=\frac{\left(k \beta \mathrm{e}^{-m \tau}-a p\right) N_{n}^{u}}{a p}, \\
& N_{n}^{v}=\frac{k\left(k \beta \mathrm{e}^{-m \tau}-a p\right) N_{n}^{u}}{a p^{2}} .
\end{aligned}
$$

It is readily seen that

$$
N_{n}^{u} \leq \underline{u} \leq \bar{u} \leq M_{n}^{u}, \quad N_{n}^{w} \leq \underline{w} \leq \bar{w} \leq M_{n}^{w}, \quad N_{n}^{v} \leq \underline{v} \leq \bar{v} \leq M_{n}^{v} .
$$

The sequences $M_{n}^{u}, M_{n}^{w}$, and $M_{n}^{v}$ are nonincreasing and the sequences $N_{n}^{u}, N_{n}^{w}$, and $N_{n}^{v}$ are nondecreasing.

To prove the monotonicity of $N_{n}^{u}$ and $M_{n}^{u}$, we follow the ideas of Uh Zapata et al. [6]; consider $R_{0}=\beta k \mathrm{e}^{-m \tau} / a p$ and the following expressions for $N^{u}, N^{w}, N^{v}, M^{u}, M^{w}$, and $M^{v}$

$$
\begin{array}{cl}
M_{n}^{u}=\frac{L\left(M_{n-1}^{u}+M_{n-1}^{w}\right)}{d\left(M_{n-1}^{u}+M_{n-1}^{w}\right)+\beta N_{n-1}}, & M_{n}^{w}=\left(R_{0}-1\right) M_{n}^{u}, \quad M_{n}^{v}=\frac{k}{p}\left(R_{0}-1\right) M_{n}^{u} \\
N_{n}^{u}=\frac{L\left(N_{n-1}^{u}+N_{n-1}^{w}\right)}{d\left(N_{n-1}^{u}+N_{n-1}^{w}\right)+\beta M_{n}^{v}}, \quad N_{n}^{w}=\left(R_{0}-1\right) N_{n}^{u}, \quad N_{n}^{v}=\frac{k}{p}\left(R_{0}-1\right) N_{n}^{u} .
\end{array}
$$

We prove the result by induction so we first show that $M_{2}^{u}-M_{1}^{u} \leq 0$,

$$
M_{2}^{u}-M_{1}^{u}=\frac{L \mathrm{e}^{-m \tau} M_{1}^{u}}{d \mathrm{e} M_{1}^{u}+a\left(R_{0}-1\right) N_{1}^{u}}-\frac{L}{d} \leq \frac{L}{d}-\frac{L}{d}=0
$$

and $N_{2}^{u}-N_{1}^{u}>0$,

$$
\begin{aligned}
N_{2}^{u}-N_{1}^{u} & =\frac{L R_{0} N_{1}}{d R_{0} N_{1}^{u}+(\beta h / p)\left(R_{0}-1\right) M_{2}^{u}}-N_{1}=N_{1}^{u}\left(\frac{L R_{0}-d R_{0} N_{1}^{u}-(\beta h / p)\left(R_{0}-1\right) M_{2}^{u}}{d R_{0} N_{1}^{u}+(\beta h / p)\left(R_{0}-1\right) M_{2}^{u}}\right) \\
& =\frac{N_{1}^{u}}{d R_{0} N_{1}^{u}+(\beta h / p)\left(R_{0}-1\right) M_{2}^{u}}\left(L R_{0}-L R_{0}+\beta R_{0} M_{1}^{v}-\frac{h \beta}{p}\left(R_{0}-1\right) M_{2}^{u}\right) \\
& =\frac{N_{1}^{u}}{d R_{0} N_{1}^{u}+(\beta h / p)\left(R_{0}-1\right) M_{2}^{u}}\left(\frac{\beta h}{p} R_{0}\left(R_{0}-1\right) M_{1}^{u}-\frac{h \beta}{p}\left(R_{0}-1\right) M_{2}^{u}\right) \\
& =\frac{\beta h\left(R_{0}-1\right) N_{1}^{u}}{p\left(d R_{0} N_{1}^{u}+(\beta h / p)\left(R_{0}-1\right) M_{2}^{u}\right)}\left(R_{0} M_{1}^{u}-M_{2}^{u}\right) \\
& >\frac{\beta h\left(R_{0}-1\right) N_{1}^{u}}{p\left(d R_{0} N_{1}^{u}+(\beta h / p)\left(R_{0}-1\right) M_{2}^{u}\right)}\left(R_{0} M_{1}^{u}-M_{2}^{u}\right)>0 .
\end{aligned}
$$


For the next step consider the function $f(x)=a x /(b x+c)$, with $a, b$, and $c$ positive, which is monotone increasing. The induction hypothesis is $M_{n}^{u} \leq M_{n-1}^{u}$ and $N_{n}^{u} \geq N_{n-1}^{u}$,

$$
M_{n+1}^{u}=\frac{L R_{0} M_{n}^{u}}{d R_{0} M_{n}^{u}+\beta N_{n}^{u}}=\frac{L R_{0} M_{n}^{u}}{d R_{0} M_{n}^{u}+(\beta h / p)\left(R_{0}-1\right) N_{n}^{u}} .
$$

The last function is increasing and by the induction hypothesis we have

$$
M_{n+1}^{u} \leq \frac{L R_{0} M_{n-1}^{u}}{d R_{0} M_{n-1}^{u}+(\beta h / p)\left(R_{0}-1\right) N_{n}^{u}} \leq \frac{L R_{0} M_{n-1}^{u}}{d R_{0} M_{n-1}^{u}+(\beta h / p)\left(R_{0}-1\right) N_{n}^{u}}=M_{n}^{u}
$$

therefore the sequence $M_{n}^{u}$ is nonincreasing. For the sequence $N_{n}^{u}$ we use similar ideas and the behaviour of the sequence $M_{n}^{u}$ just proved. One has

$$
N_{n+1}^{u}=\frac{L R_{0} N_{n}^{u}}{L R_{0} N_{n}^{u}+\beta M_{n+1}^{v}}=\frac{L R_{0} N_{n}^{u}}{L R_{0} N_{n}^{u}+(\beta h / p)\left(R_{0}-1\right) M_{n+1}^{u}} .
$$

The last function is increasing and by the induction hypothesis we have

$$
N_{n+1}^{u} \geq \frac{L R_{0} N_{n-1}}{L R_{0} N_{n-1}+(\beta h / p)\left(R_{0}-1\right) M_{n+1}^{u}} \geq \frac{L R_{0} N_{n-1}}{L R_{0} N_{n-1}+(\beta h / p)\left(R_{0}-1\right) M_{n}^{u}}=N_{n}^{u},
$$

therefore the sequence $N_{n}^{u}$ is nondecreasing. The behaviour for the sequences $N_{n}^{w}, N_{n}^{v}, M_{n}^{w}$, and $M_{n}^{v}$ follows from the nonincreasing sequence $M_{n}^{u}$ and the nondecreasing sequence $N_{n}^{u}$.

Hence, the limit of each sequence in $N_{n}^{u}, N_{n}^{w}, N_{n}^{v}, M_{n}^{u}, M_{n}^{w}$, and $M_{n}^{v}$ exists. Denote

$$
\begin{array}{ll}
\bar{x}=\lim _{n \rightarrow \infty} M_{n}^{u}, & \underline{x}=\lim _{n \rightarrow \infty} N_{n}^{u}, \\
\bar{y}=\lim _{n \rightarrow \infty} M_{n}^{w}, & \underline{y}=\lim _{n \rightarrow \infty} N_{n}^{w}, \\
\bar{z}=\lim _{n \rightarrow \infty} M_{n}^{v}, & \underline{z}=\lim _{n \rightarrow \infty} N_{n}^{v} .
\end{array}
$$

We therefore obtain from (4.57) and (4.66) that

$$
(\bar{x}-\underline{x})\left[\frac{d k \beta \mathrm{e}^{-m \tau}}{a p}-\frac{k \beta\left(k \beta \mathrm{e}^{-m \tau}\right)-a p}{a p^{2}}\right]=0 .
$$


Noting that (H1) holds and $R_{0}>1$, it follows that

$$
\frac{d k \beta \mathrm{e}^{-m \tau}}{a p}>\frac{k \beta\left(k \beta \mathrm{e}^{-m \tau}-a p\right)}{a p^{2}},
$$

which together with the previous equation yields $\bar{x}=\underline{x}$. We therefore derive from (4.57) $y$ (4.66) that $\bar{y}=\underline{y}, \bar{z}=\underline{z}$. Noting that if $R_{0}>1$, by Theorem 3.1, the virus-infected equilibrium $E_{2}$ is locally asymptotically stable, and if in addition (H1) holds, we conclude that $E_{2}$ is globally asymptotically stable.

Now we prove the global stability for the disease-free equilibrium

Theorem 4.3. If $R_{0}<1$ the disease-free equilibrium $E_{1}(L / d, 0,0)$ of $(1.3)$ is globally asymptotically stable.

Proof. Let $(u(x, t), w(x, t), v(x, t))$ be a solution to problem with $\phi_{i}(x, 0) \neq 0,(i=1,2,3)$. We have $u(x, t)>0, w(x, t)>0$, and $v(x, t)>0$ for all $x \in \bar{\Omega}$. Let $\left(u^{(1)}(x, t), w^{(1)}(x, t), v^{(1)}(x, t)\right)$ be a solution to the following problem:

$$
\begin{gathered}
\frac{\partial u^{(1)}}{\partial t}=L-d u^{(1)}(x, t), \\
\frac{\partial w^{(1)}}{\partial t}=\frac{\beta \mathrm{e}^{-m \tau} u^{(1)}(x, t-\tau) v^{(1)}(x, t-\tau)}{u^{(1)}(x, t-\tau)+w^{(1)}(x, t-\tau)}-a w^{(1)}(x, t), \\
\frac{\partial v^{(1)}}{\partial t}=D \Delta v^{(1)}(x, t)+k w^{(1)}(x, t)-p v^{(1)}(x, t) .
\end{gathered}
$$

Therefore for $t>0, x \in \bar{\Omega}$ we have

$$
0 \leq u(x, t) \leq u^{(1)}(x, t), \quad 0 \leq w(x, t) \leq w^{(1)}(x, t), \quad 0 \leq v(x, t) \leq v^{(1)}(x, t) .
$$

We derive from the first equation that

$$
\lim _{t \rightarrow \infty} u^{(1)}(x, t)=\frac{L}{d}
$$

so we can conclude

$$
\limsup _{t \rightarrow \infty} \max u(x, t) \leq \frac{L}{d}
$$



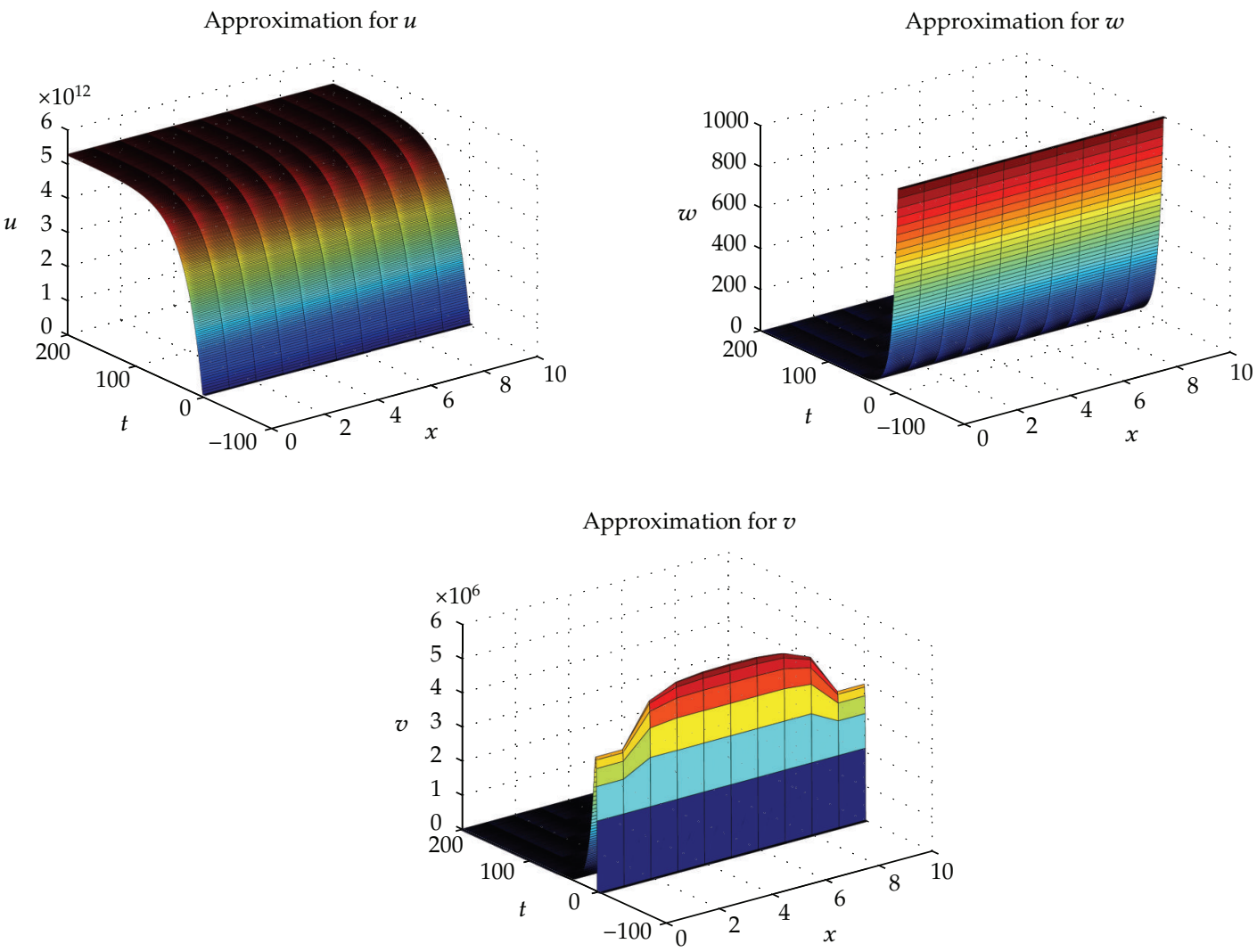

Figure 1: Simulations with parameters $L=2^{11}, d=3.78^{-2}, a=3.38 d, p=0.67, \beta=1.45^{-6}, k=5.18^{3}, m=0.2$, and $D=0.5 . R_{0}=0.039426$.

Hence, for $\epsilon>0$ sufficiently small, there exists a $t_{1}$ such that $u^{(1)}(x, t) \leq L / d+\epsilon$ for all $x \in \bar{\Omega}$ and $t \geq t_{1}$. Hence, $(w(x, t), v(x, t))$ is a lower solution to the following problem:

$$
\begin{gathered}
\frac{\partial \omega_{2}^{(1)}}{\partial t}=\frac{\beta \mathrm{e}^{(-m \tau)}(L / d+\epsilon) v^{(1)}(x, t-\tau)}{L / d+\epsilon+\omega_{2}^{(1)}(x, t-\tau)}-a \omega_{2}^{(1)}(x, t), \\
\frac{\partial \omega_{3}^{(1)}}{\partial t}=D \Delta \omega_{3}^{(1)}+k \omega_{2}^{(1)}(x, t)-p \omega_{3}^{(1)}(x, t), \\
\frac{\partial \omega_{2}^{(1)}}{\partial \eta}=\frac{\partial \omega_{3}^{(1)}}{\partial \eta}=0, \quad t>t_{1} ; x \in \partial \widehat{\Omega}, \\
\omega_{2}^{(1)}(x, t)=w(x, t), \quad \omega_{3}^{(1)}(x, t)=v(x, t), \quad t \in\left[-\tau, t_{1}\right], x \in \bar{\Omega} .
\end{gathered}
$$



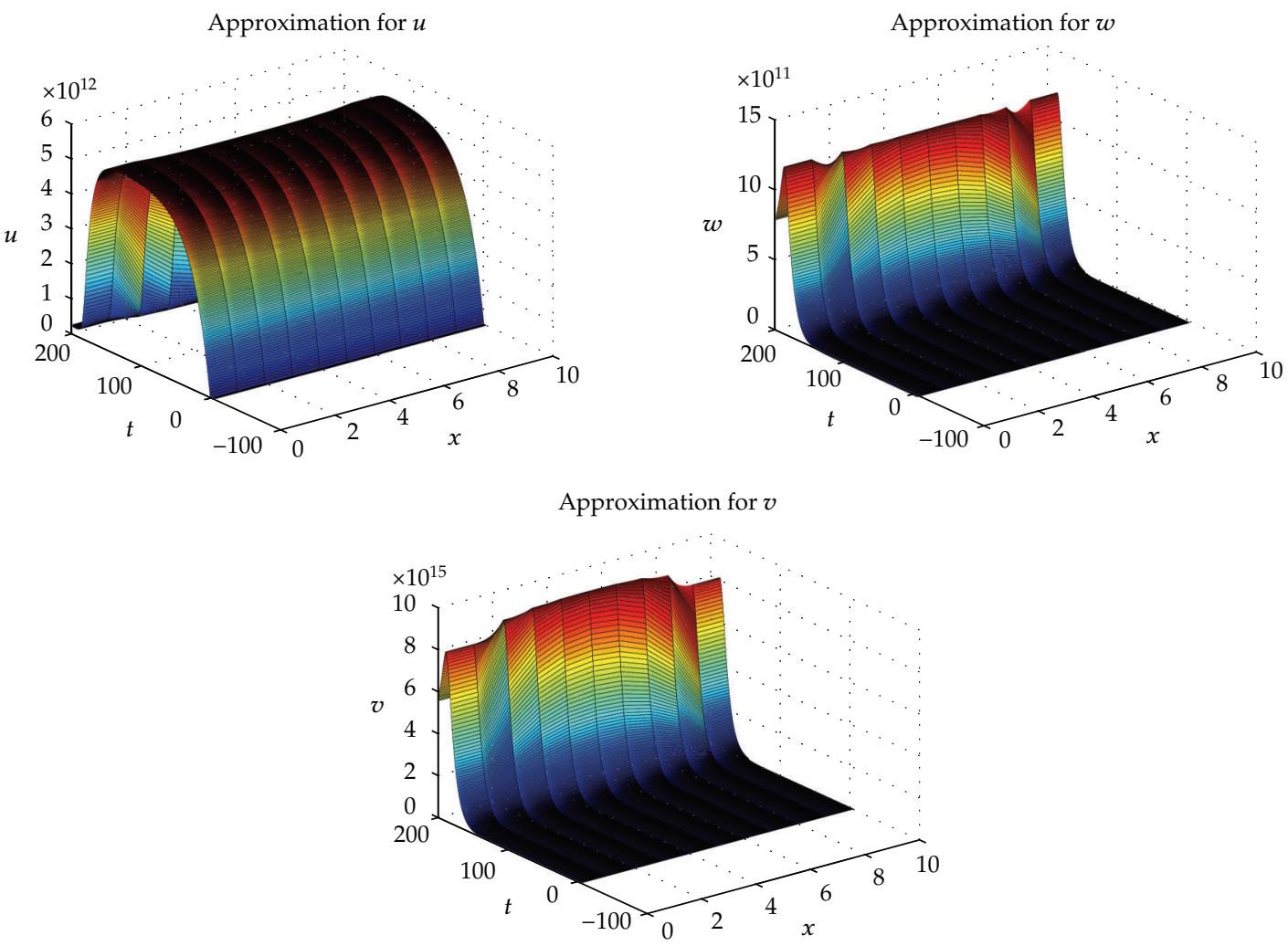

Figure 2: Simulations with parameters $L=2^{11}, d=3.78^{-2}, a=3.38 d, p=0.67, \beta=1.45^{-4}, k=5.18 \times 10^{3}$, $m=0.2$, and $D=0.1 . R_{0}=3.9426$.

Consider $\left(u_{2}(t), u_{3}(t)\right)$ as the solution for

$$
\begin{gathered}
\dot{u}_{2}=\frac{\beta \mathrm{e}^{-m \tau}(L+\epsilon) u_{3}(t-\tau)}{L / d+\epsilon+u_{2}(t-\tau)}-a u_{2}(t), \\
\dot{u}_{3}=k u_{2}(t)-p u_{3}(t), \\
u_{2}(t)=\max _{x \in \bar{\Omega}} w(x, t), \quad u_{3}(t)=\max _{x \in \bar{\Omega}} v(x, t), \quad t \in\left[-\tau, t_{1}\right] .
\end{gathered}
$$

Then with $R_{0}<1$ according to Lemma 4.1 we have that

$$
\lim _{t \rightarrow \infty} u_{2}(t)=0, \quad \lim _{t \rightarrow \infty} u_{3}(t)=0 .
$$

By comparison, it follows that

$$
\lim _{t \rightarrow \infty} w(t)=0, \quad \lim _{t \rightarrow \infty} v(t)=0
$$




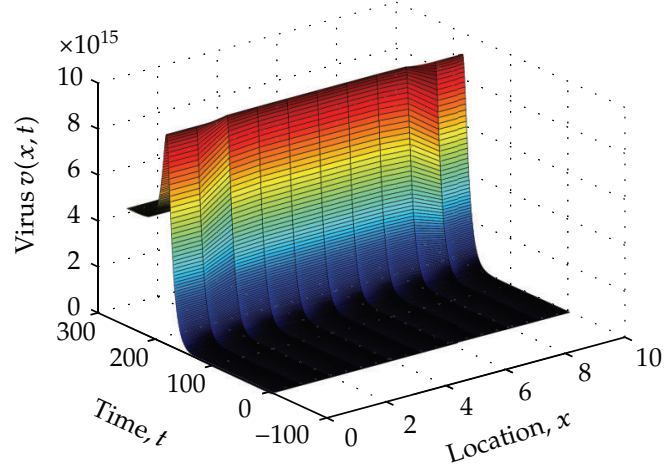

(a) $D=0.01, \tau=4$

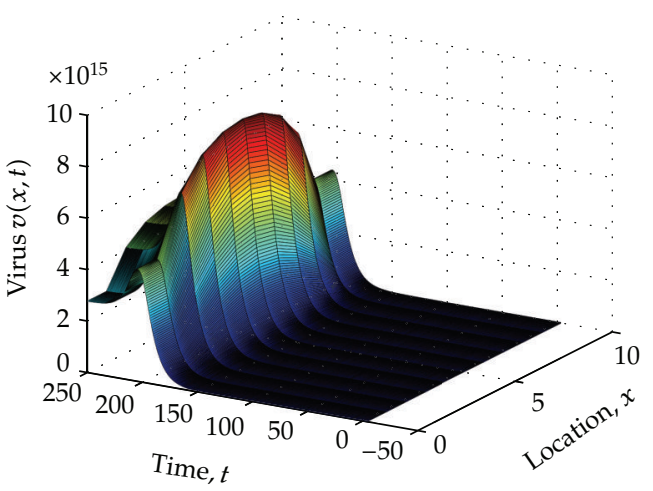

(c) $D=1, \tau=4$

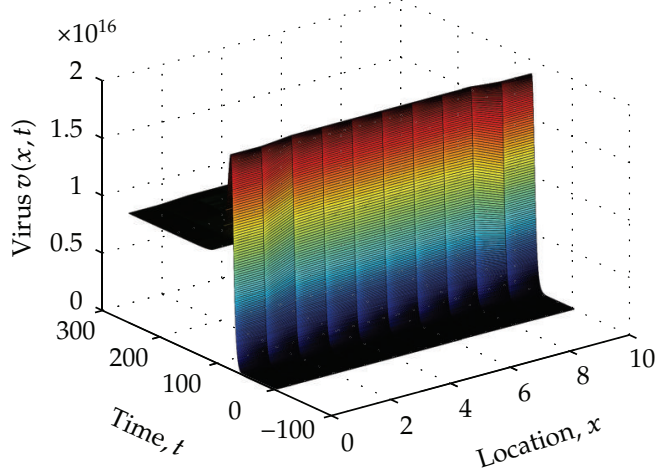

(b) $D=0.01, \tau=0.9$

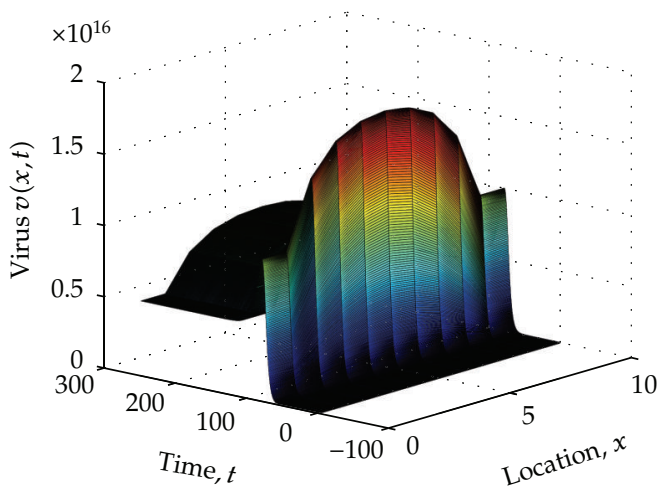

(d) $D=1, \tau=0.9$

Figure 3: In this case the parameters are $L=2^{11}, d=3.78^{-2}, a=3.38 d, p=0.67, \beta=1.45^{-4}, k=5.18^{3}$ and $m=0.2$.

uniformly for $x \in \bar{\Omega}$. Hence, for $\epsilon>0$ sufficiently small, by comparison there is a $t_{2} \geq t_{1}$ such that if $t \geq t_{2}, w(x, t)<\epsilon, v(x, t)<\epsilon$ for all $x \in \bar{\Omega}$ and $t \geq t_{2}$.

As in the proof of Theorem $4.2 u(x, t)$ is an upper solution for the following problem:

$$
\begin{gathered}
\partial \omega_{1}^{(1)}=L-d \omega_{1}^{(1)}-\beta \omega_{1}^{(1)} \epsilon \\
\frac{\partial \omega_{1}^{(1)}}{\partial \eta}=0, \quad t \geq t_{2}, x \in \bar{\Omega}, \quad \omega_{1}^{(1)}\left(x, t_{2}\right)=\frac{1}{2} u\left(x, t_{2}\right), \quad x \in \bar{\Omega}
\end{gathered}
$$

from the above equation we have that

$$
\lim _{t \rightarrow \infty} \omega_{1}^{(1)}(x, t)=\frac{L}{d+\beta \epsilon}
$$




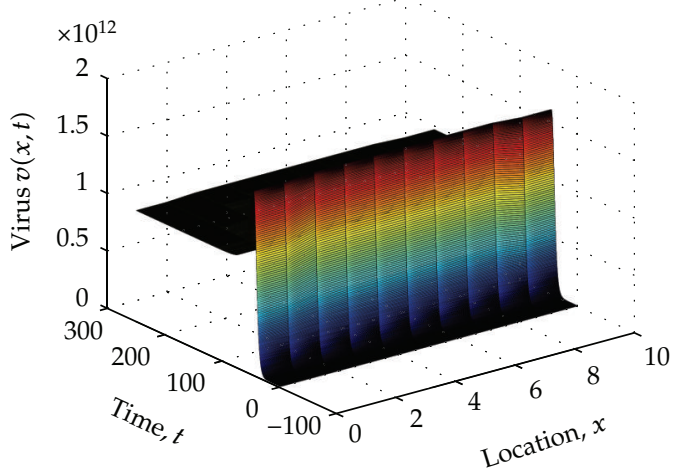

(a) $D=0.01, \tau=0.9$

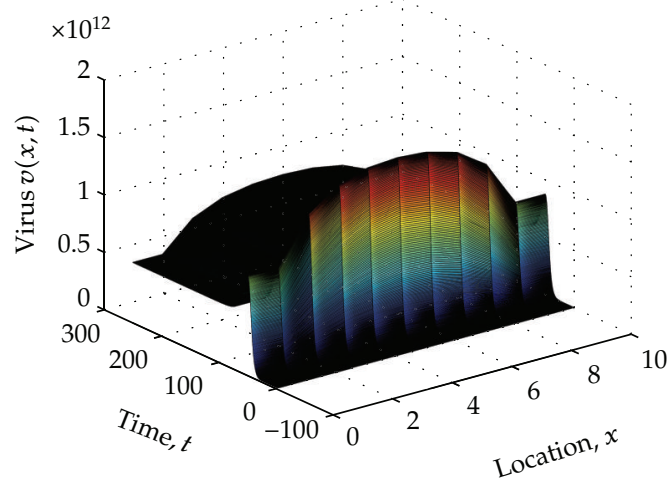

(c) $D=1, \tau=0.9$

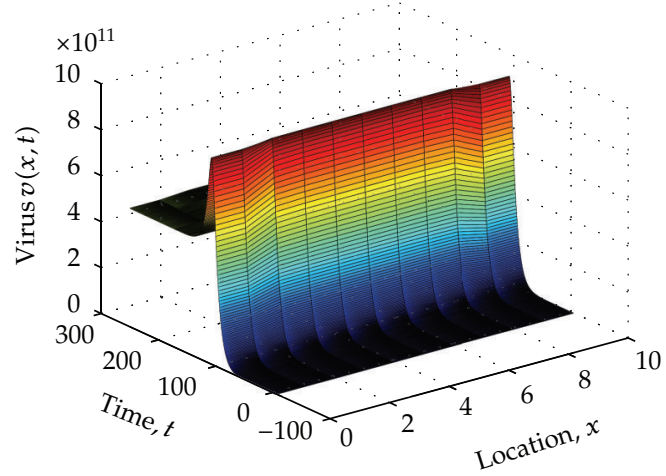

(b) $D=0.01, \tau=4$

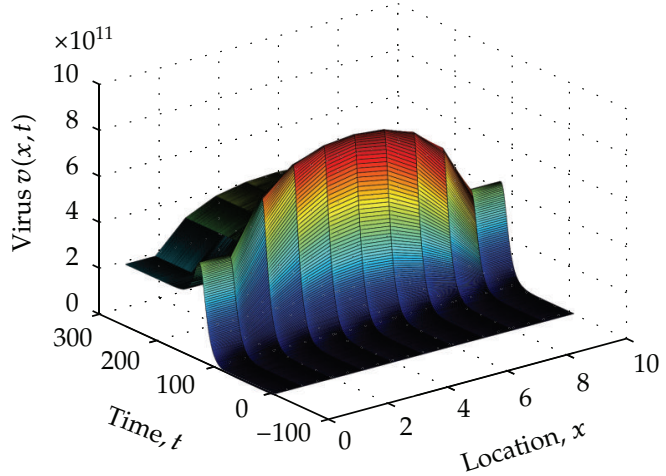

(d) $D=1, \tau=4$

Figure 4: In this case the parameter are $L=2^{7}, d=3.78^{-2}, a=3.38 d, p=0.67, \beta=1.45^{-4}, k=5.18^{3}$ and $m=0.2$.

uniformly for $x \in \bar{\Omega}$. Since this holds for arbitrary $\epsilon>0$ sufficiently small, by comparison we conclude that

$$
\liminf _{t \rightarrow \infty} \min u(x, t) \geq \frac{L}{d}
$$

which together with (4.72) gives

$$
\lim _{t \rightarrow \infty} u(x, t)=\frac{L}{d}
$$

uniformly for $x \in \bar{\Omega}$. We already have by Theorem 3.1 that the disease-free equilibrium $E_{1}$ is locally asymptotically stable. And now we have proved that it is also globally asymptotically stable. 


\section{Numerical Simulations}

In this section we illustrate some numerical solutions for systems (1.3). In the numerical simulation display in Figure 1 we illustrate the stability for the disease-free equilibrium according to Theorem 3.1. In this case the basic reproductive number is $R_{0}=0.039426$. In the graphics we see how the level of uninfected cells increases from the initial condition and the number of infected cells and virus in the body goes to zero.

In Figure 2 consider the case $R_{0}>1$ in this case we consider a bigger rate of infection for the cells in the graphics we see how the number of infected cells and viruses increases when the time passes, and when the number of susceptible cells decreases the number of virus also decreases to the value $v^{*}$.

Now in Figure 3 we just show the level of virus in different for different values of the diffusion constant $D$ and the delay $\tau$. We see that a bigger delay increases the time needed for the virus to reach the value $v^{*}$, meanwhile a mayor value for the constant $D$ just affects the levels of the virus according to the space and does not affect significantly the time needed for the virus to reach $v^{*}$. In Figure 4 we consider a lower value for $\lambda$, which is the uninfected cell production rate. In this case we see how the time to reach the value $v^{*}$ of the endemic equilibrium is lower and again the diffusion rate has no significant effect on the time; its effect is on the level of virus in the system. The delay is what really affect the time to reach the value $v^{*}$.

\section{Acknowledgments}

This paper was supported by UADY under Grant UADY-CA-34 and Mexican SNI under Grants 15284 and 33365.

\section{References}

[1] M. A. Nowak, S. Bonhoeffer, A. M. Hill, R. Boheme, H. C. Thomas, and H. McDade, “Viral dynamics in hepatitis B virus infection," Proceedings of the National Academy of Sciences of the United States of America, vol. 93, no. 9, pp. 4398-4402, 1996.

[2] X. Tian and R. Xu, "Asymptotic properties of a hepatitis B virus infection model with time delay," Discrete Dynamics in Nature and Society, vol. 2010, Article ID 182340, 21 pages, 2010.

[3] K. Wang and W. Wang, "Propagation of HBV with spatial dependence," Mathematical Biosciences, vol. 210, no. 1, pp. 78-95, 2007.

[4] R. Xu and Z. Ma, "HBV model with diffusion and time delay," Journal of Theoretical Biology, vol. 257, pp. 499-509, 2009.

[5] R. Redlinger, "Existence theorems for semilinear parabolic systems with functionals," Nonlinear Analysis. Theory, Methods \& Applications, vol. 8, no. 6, pp. 667-682, 1984.

[6] M. Uh Zapata, E. Avila Vales, and A. G. Estrella, "Asymptotic behavior of a competition-diffusion system with variable coefficients and time delays," Journal of Applied Mathematics, vol. 2008, Article ID 537284, 17 pages, 2008. 




Advances in

Operations Research



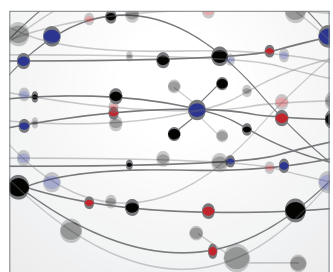

\section{The Scientific} World Journal
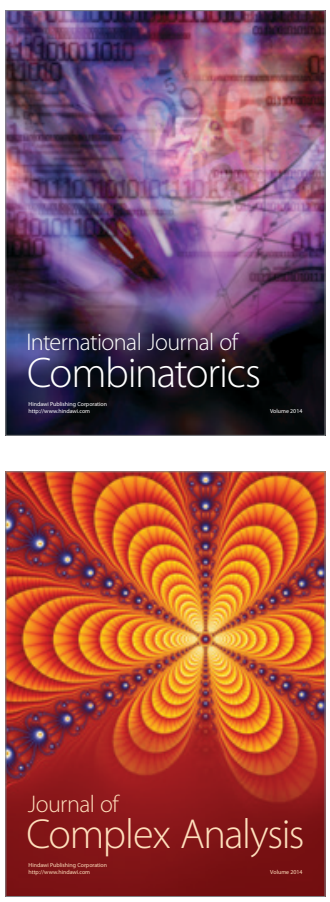

International Journal of

Mathematics and

Mathematical

Sciences
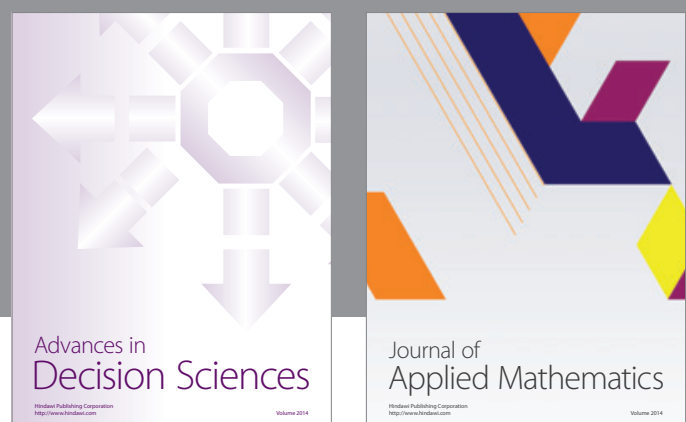

Journal of

Applied Mathematics
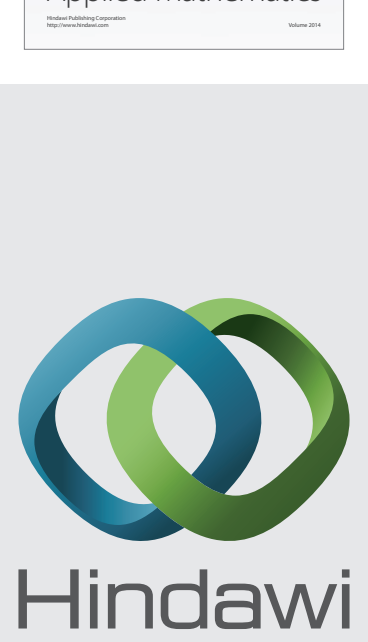

Submit your manuscripts at http://www.hindawi.com
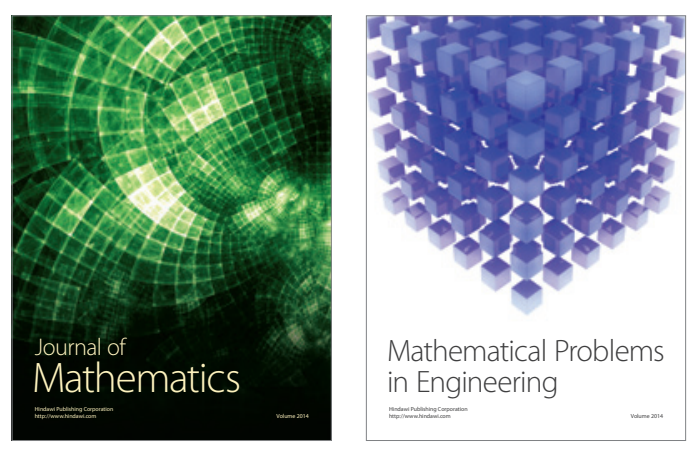

Mathematical Problems in Engineering
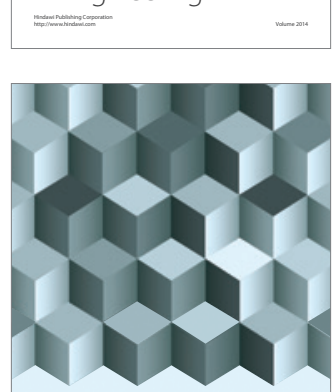

Journal of

Function Spaces
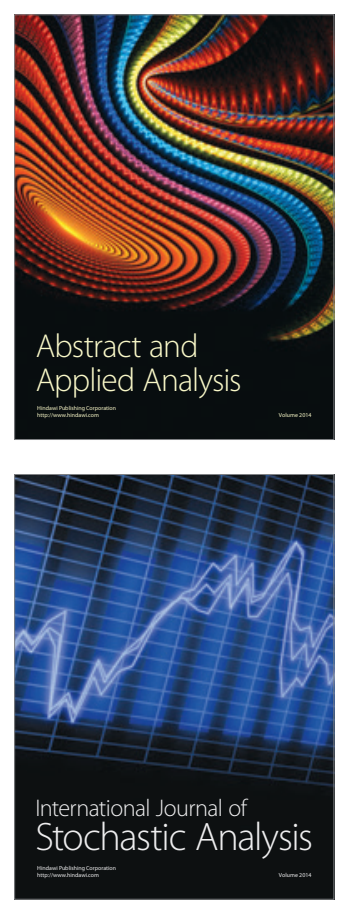

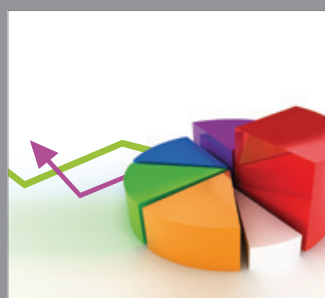

ournal of

Probability and Statistics

Promensencen
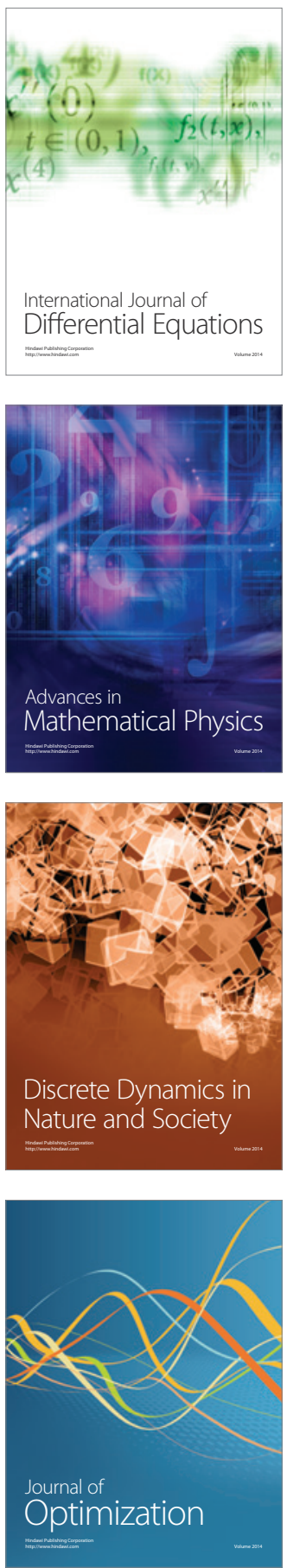